\title{
Discovery of Fuzzy Hierarchical Association Rules
}

\author{
Reena Kumari \\ Research Scholar \\ Department of Computer Science \& Engineering \\ GJUST ,Hisar
}

\author{
Jyoti Vashishtha \\ Assistant Professor \\ Department of Computer Science \& Engineering \\ GJUST,Hisar
}

\begin{abstract}
A number of techniques have been developed to turn data into useful knowledge. Most of the algorithms in data mining find association rules among transactions using binary values and at single concept level. However it will be more exciting to discover hierarchical association rules for decision makers. In this work we have integrated association rule mining with fuzzy set theory and hierarchy. We have proposed an algorithm to discover hierarchical fuzzy association rules. We have used different minimum support and membership functions at each level of hierarchy. We have also used a predefined taxonomy for multilevel of hierarchy.
\end{abstract}

\section{General Terms}

Association Rule, Fuzzy Set.

\section{Keywords}

Hierarchical Fuzzy Association Rules, Taxonomy, Membership function

\section{INTRODUCTION}

An approach that converts data into knowledge is known as data mining. Association rule mining is one of the very important tasks in data mining. It can be applied in different areas like marketing, business planning etc. For example, the association rules can be used to identify the customer buying habits in a market-basket analysis, like "if customers buy personal computer, they are more likely to buy an antivirus or printer as well". In general, every association rule must satisfy two user specified constraints called support and confidence. The support of a rule $X=>Y$ is defined as the percentage of transactions that contain $(\mathrm{X} \vee \mathrm{Y})$, where $\mathrm{X}$ and $\mathrm{Y}$ are disjoint sets of items from the given dataset. The confidence is defined as the ratio support $(\mathrm{X} \vee \mathrm{Y}) / \operatorname{support}(\mathrm{X})[1]$

Deriving association rules from transaction dataset is most commonly seen in data mining [2, 3, 4, 5, 6, 7]. Agrawal and his co-workers have proposed a number of algorithms for finding association rules $[2,3]$. The items considered in their approach have no hierarchical relationship i.e. associations are discovered at single level. So, in this paper, we are focusing on designing an algorithm to discover hierarchical fuzzy association rules. Mining hierarchical fuzzy association rules will lead to discovery of more general, accurate, exact and important knowledge from data. Fuzzy set theory [8], firstly proposed by

Zadeh and Goguen, is being used more and more frequently in intelligent systems because of its simplicity and similarity to human reasoning [9]. In this paper, we are using Han and Fu's encoding scheme [10] to represent node in predefined taxonomy and then finding interesting fuzzy rules by modifying Srikant and Agrawal's method [11] in order to manage hierarchical data.

\section{RELATED WORK}

Agrawal et al. have proposed several mining algorithms for finding association rules in transaction data based on the concept of large itemsets [12]. It is an AIS algorithm known to be the first published algorithm to generate all large itemsets in a transaction database. The AIS algorithm makes multiple passes over the entire database and in each pass, it scans all transactions. In the first pass, it counts the support of individual items and determines which of them are large or frequent in the database. Large itemsets of each pass are extended to generate candidate itemsets. After scanning a transaction, the common itemsets among large itemsets of the previous pass and items of this transaction are determined. These common itemsets are extended with other items in the transaction to generate new candidate itemsets. A large itemset ' $l$ ' is extended with only those items in the transaction that are large and occur in the lexicographic ordering of items. It uses an estimation tool and pruning technique to perform the task efficiently. The estimation and pruning techniques determine candidate sets by omitting unnecessary itemsets from the candidate sets. Then, the support of each candidate set is computed. Candidate sets having supports greater than or equal to min support are chosen as large itemsets. These large itemsets are extended to generate candidate sets for the next pass. This process terminates when no more large itemsets are found. The disadvantage of this technique is the unnecessarily generation and counting of too many small candidate itemsets.

They also proposed a method for mining association rules from data sets using quantitative and categorical attributes [13]. This method determines the number of partitions for each quantitative attribute and then maps all possible values of each attribute onto a set of consecutive integers. Further, a number of methods have been proposed to handle numeric attributes and to derive association rules. Apriori and AprioriTid algorithms [5] are used to discover association rules between items in a large database of sales transactions. The results reveal that these algorithms always outperform the earlier algorithms AIS and SETM. Studies on mining association rules find rules at single concept level; however, mining association rules at multiple concept levels may lead to the discovery of more specific and concrete knowledge from data. In this study, Han, J., and Fu, Y. developed a topdown progressive deepening method for mining multiple level association rules from large transaction databases [14]. Srikant R. and Agarwal R. discussed concept hierarchy handling, methods for mining flexible multiple-level association rules and adaptation to difference mining requests in their studies [15, 16]. They introduced the problem of mining generalized association rules where a database of transactions consists of a set of items and taxonomy (is-ahierarchy) on the items. Their method finds associations between items at any level of the taxonomy. Wong, M.H. et 
al. proposed a method to handle quantitative attributes for which each attribute is assigned several fuzzy sets [17]. Fuzzy sets handle numerical values better than existing methods because fuzzy sets soften the effect of sharp boundaries. The fuzzy set concept is better than the partition method because fuzzy sets provide a smooth transition between member and non-member of a set.

In many real life applications, the related taxonomic structures may not be necessarily crisp, rather certain fuzzy taxonomic structures reflecting partial belonging of one item to another may pertain. For example, Carrot may be regarded as being both Fruit and Vegetable, although to different degrees. In this example, a sub-item belongs to its super-item with a certain degree and a crisp taxonomic structure assumes that the child item belongs to its ancestor with degree 1 . However, in a fuzzy taxonomy; this assumption is no longer true. Different degrees may pertain across all nodes (item sets) of the structure. Chen, G. et al. focus on the issue of mining generalized association rules with fuzzy taxonomic structures [18]. They extend Apriori and Fast algorithm to allow discovering the relationships between data attributes upon all levels of fuzzy taxonomic structures.

Lee et al. are the first in relating fuzzy sets and association rules. In their work, fuzzy sets are introduced to diminish the granularity of quantitative attributes [19]. The model uses a membership threshold to change fuzzy transactions into crisp ones before looking for ordinary association rules in the set of crisp transactions. Gautam and Pardasani introduced a novel algorithm for generating fuzzy multilevel association rule [20]. The algorithm is based on the concept that the larger number of items purchased in a transaction means the lower level of association among the items in the transaction. The proposed fuzzy mining algorithm can thus generate large itemsets level by level and then derive fuzzy association rules from transaction dataset. According to Rountree et al. if a single minimum support threshold is used and is set high, those association rules involving rare items will not be discovered [21]. Use of a single and lower minimum support threshold, on the other hand, would result in too many uninteresting association rules. Usha Rani et al. propose a multilevel association rule mining using fuzzy concepts [22]. They employ different fuzzy membership function to retrieve efficient association rules from multi level hierarchies that exist in a transaction dataset. They use the fuzzy-set concepts to retrieve multilevel association rules. There approach adopts a top-down progress and also incorporates fuzzy boundaries instead of sharp boundary intervals to derive large itemsets. Hong et al. designed a fuzzy multilevel mining algorithm that can process transaction data with quantitative values and discover interesting patterns among them[23]. The rules thus mined exhibit quantitative regularity on multiple levels and can be used to provide suggestions to appropriate supervisors. Prakash et.al. describe that association rules are based on the preset minimum support threshold given by domain experts [24]. The accuracy in setting up this threshold directly influences the number and quality of the association rules discovered. According to them the number of association rules is large, some interesting rules will be missing and the rules quality requires further analysis and decision making using these rules could lead to risky actions. So they focus on mining both the frequent and infrequent association rules which are more interesting and do not have redundant rules and compare single level and multi level association rule mining. Generalized association rules will be obtained if single level mining is performed. These rules can only help in very high level decision making. In order to allow for in-depth decision making, more specific association rules are obtained.

\section{NOTATION}

$\begin{array}{ll}\text { The following notation is used in our proposed algorithm: } \\ \mathrm{n} & \text { the number of transactions } \\ \mathrm{D}_{i} & \text { the } i \text { th transaction, } \\ \mathrm{R}_{j l} & \text { the } l \text { th fuzzy region of } \mathrm{I}_{\mathrm{j}} ; \\ \mathrm{V}_{i j} & \text { the quantitative value of } \mathrm{I}_{\mathrm{j}} \text { in } \mathrm{Di} \\ \mathrm{F}_{i j} & \text { the fuzzy set converted from } \mathrm{Vij} \\ \mathrm{Count}_{j l} & \text { the summation of Fij values for } \mathrm{i}=1 \text { to } \mathrm{n} \\ \alpha_{L}^{i} & \text { the predefined minimum support threshold } \\ \beta & \text { where } \mathrm{L} \text { denotes level and } \mathrm{i} \in\{1,2,3\} \\ \mathrm{Cr} & \text { the predefined minimum confidence threshold } \\ \mathrm{Lr} & \text { the set of candidate itemsets with } \mathrm{r} \text { items }\end{array}$

\section{THE PROPOSED MODEL}

The proposed algorithm works on quantitative data and finds all the large itemsets for the given transactions by comparing the fuzzy count of each candidate itemset with its support threshold. It uses different minimum support at each level. Furthermore, some pruning strategies are used to reduce the number of candidate itemsets generated. The desired algorithm is given step by step in figure 1 .

\section{AN ILLUSTRATIVE EXAMPLE}

An illustrative example is given to understand well the concept of the proposed model and algorithm and how the process of the generating fuzzy association rule mining is performed step by step. We are using taxonomy of figure 4 . We are using function $f(x)$ as membership function at each level. But value of $a, b$ and $m$ are different at each level.

$$
\mathrm{f}(\mathrm{x})=\left\{\begin{array}{l}
0, \quad \mathrm{x}<0 \text { or } x>b \\
\frac{(x-a)}{(b-a)}, \quad x \geq a, x<m \\
\frac{(b-x)}{(b-m)}, x \geq m, x \leq b
\end{array}\right.
$$

There are 3 regions namely low,middle, high at each level. For level 1 values of $a, b, m$ at each region is

$$
\left\{\begin{array}{c}
\text { low } a=0, b=11, m=6 \\
\text { mid } a=6, b=21, m=11 \\
\text { high } a=11, b=30, m=21
\end{array}\right.
$$


Input:- A body of $\mathrm{n}$ quantitative transaction data, a set of membership functions, predefined taxonomy,predefined minimum support values $\quad \alpha{ }_{\mathrm{L}}^{\mathrm{m}}$ a predefined confidence value $\beta$, where $\mathrm{m} \in\{1,2,3\}$.

Output:-A set of hierarchical fuzzy association rules.

Step -1: Encode taxonomy using a sequence of numbers and the symbol “*”,

Step -2: Set $L=1$, where $L$ is used to store the level number being processed whereas $L \in\{1,2,3\}$ (as we consider up to 3-levels of hierarchies).

Step-3: Group the items with the same first L digits in each transaction T, and add the occurrence of the items in the same groups in $\mathrm{Vij}$.

Step -4: Transform the quantitative value $V_{i j}$ of each transaction datum $D_{i}(i=1$ to $n$ ) into a fuzzy set using the given membership functions. Denote fuzzy values by $\mathrm{F}_{\mathrm{ijk}}$.

Step -5: Calculate the scalar cardinality of each fuzzy region $R j 1$ in the transaction data: countjl $=\sum \mathrm{F}_{\mathrm{ijk}}$.

Step -6: Check whether the value countjl is larger than or predefined minimum support value. If it is equal to or greater than the minimum support value, put it in the large 1-itemsets $\left(\mathrm{L}_{1}\right)$.

Step-7: Generate the candidate set $\mathrm{C}_{2}$ from $\mathrm{L}_{1}$.

Step-8: For each newly formed candidate 2-itemset $\mathrm{s}$ with items $\left(\mathrm{s}_{1} ; \mathrm{s}_{2}\right)$ in $\mathrm{C}_{2}$ :

(a) Calculate the fuzzy value of $s$ in each transaction datum $D_{i}$ as $F_{\text {is }}=F_{\text {is } 1} \cap F_{\text {is } 2}$, where $F_{\text {isj }}$ is the membership value of $\mathrm{D}_{\mathrm{i}}$.

(b) Calculate the scalar cardinality of $s$ in the transaction data as counts $=\sum F_{\text {is }}$.

(c) If counts is larger than or equal to the predefined minimum support value, put $\mathrm{s}$ in $\mathrm{L}_{2}$.

Step -9: IF $\mathrm{L}_{2}$ is null, then exit the algorithm; otherwise, do the next step.

Step -10: Set $r=2$, where $r$ is used to represent the number of items stored in the current large itemsets.

Step -11: Generate the candidate set $C_{r+1}$ from $L_{r}$ in a way similar to that in the apriori algorithm[13]. That is, the algorithm first joins $\mathrm{L}_{\mathrm{r}}$ and $\mathrm{L}_{\mathrm{r}}$ assuming that $\mathrm{r}-1$ items in the two itemsets are the same and the other one is different. Store in $\mathrm{C}_{\mathrm{r}+1}$ itemsets having all their sub-r-itemsets in $\mathrm{L}_{\mathrm{r}}$.

Step -12: For each newly formed $(r+1)$-itemset $\mathrm{s}$ with items $\left(\mathrm{s}_{1} ; \mathrm{s}_{2} ;::: ; \mathrm{s}_{\mathrm{r}+1}\right)$ in $\mathrm{C}_{\mathrm{r}+1}$ :

(a) Calculate the fuzzy value of $s$ in each transaction datum $D_{i}$ as $F_{\text {is }}=F_{\text {is } 1} \cap F_{\text {is } 2} \cap \cdot \cdot \cap F_{\text {isr }+1}$ where $F_{\text {isj }}$ is the membership value of $D_{i}$ in region $s_{j}$.

(b) Calculate the scalar cardinality of $\mathrm{s}$ in the transaction data as counts $=\sum \mathrm{F}_{\text {is }}$

(c) If counts is larger than or equal to the predefined minimum support value, put $s$ in $\mathrm{L}_{\mathrm{r}+1}$.

Step -13: If $L_{r+1}$ is null, then do the next step; otherwise, set $r=r+1$ and repeat Steps 10-13.

Step -14: Similar to Apriori Algorithm, confidence of an association rule mining, A => B, can be calculated by the following equation:

Where A,B $\in D$

Step -15: Keep the rules with confidence values larger than or equal to the predefined confidence threshold.

Step -16: Set $\mathrm{L}==\mathrm{L}+1$ and go to step 4(for repeating the whole processing for next level).

\section{Fig. 1: Algorithm for Fuzzy Association Rule Mining}

For level 2 values of a,b, $m$ at each region is

$$
\left\{\begin{array}{c}
\text { low } a=0, b=6, m=3 \\
\text { mid } a=3, b=18, m=14 \\
\text { high } a=14, b=22, m=18
\end{array}\right.
$$

Figure 2 shows the membership function at level 3

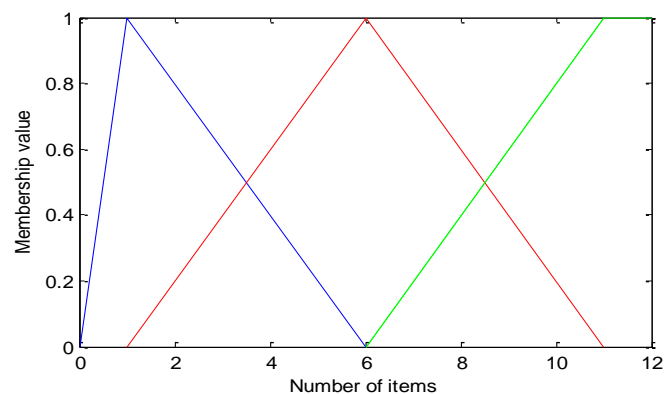

Fig. 2: Membership function

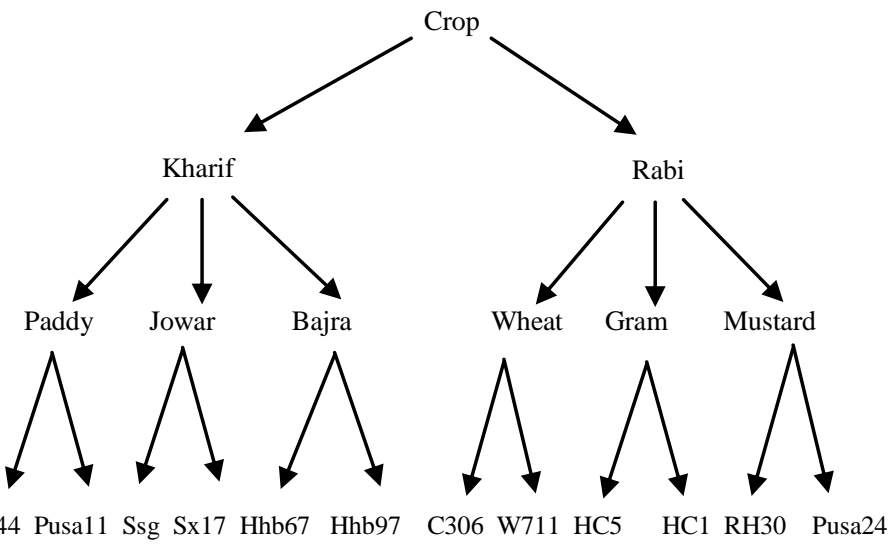

Fig. 3: Example taxonomy

For the transaction data given in table $1[\mathrm{a}]$ the proposed algorithm works as follows. 
Table 1[a]. Transaction Dataset

\begin{tabular}{|l|l|}
\hline $\begin{array}{l}\text { Trans_i } \\
\text { d }\end{array}$ & List of Items \\
\hline T1 & $(111,3),(121,4),(211,2),(221,3),(231,7),(232,2)$ \\
\hline T2 & $(112,3),(121,7),(131,7),(221,3),(231,10),(232,7)$ \\
\hline T3 & $\begin{array}{l}(112,2),(121,10),(211,5),(221,2),(231,12),(232,5 \\
)\end{array}$ \\
\hline T4 & $(121,9),(211,10),(231,9),(232,10)$ \\
\hline T5 & $(111,7),(131,8),(221,7),(231,7),(232,8)$ \\
\hline T6 & $\begin{array}{l}(112,2),(121,8),(131,10),(221,2),(231,10),(232,1 \\
0)\end{array}$ \\
\hline
\end{tabular}

Step 1: We are using encoded transaction table 1[a] and in table $1[\mathrm{~b}]$ code for each item is given.

\section{Table 1[b]. Code of Each Item}

\begin{tabular}{|l|l|l|l|}
\hline \multicolumn{2}{|c|}{$\begin{array}{c}\text { Item name } \\
\text { code terminal node) }\end{array}$} & \multicolumn{2}{c|}{ Item name } \\
code internal node)
\end{tabular}

Step 2: Now we are doing calculation for level 1 by setting $\mathrm{L}=1$.

Step 3:In table 2, we have grouped the items with the same first $\mathrm{L}$ digits in each transaction $\mathrm{T}$, and add the occurrence of the items in the same group.

\section{Table 2.Occurence of Items with same $\mathrm{L}$ digit}

\begin{tabular}{|l|l|}
\hline Trans-Id & List of Items \\
\hline T1 & $(1 * *, 5),\left(2^{* *}, 14\right)$ \\
\hline T2 & $(1 * *, 17),(2 * *, 14)$ \\
\hline T3 & $\left(1^{* *}, 12\right),(2 * *, 24)$ \\
\hline T4 & $(1 * *, 9),\left(2^{* *}, 29\right)$ \\
\hline T5 & $\left(1^{* *}, 15\right),(2 * *, 22)$ \\
\hline T6 & $(1 * *, 20),(2 * *, 22)$ \\
\hline
\end{tabular}

Step 4: We have transformed the quantities of table 2 in to fuzzy values by using triangular membership function and put them in table 3 .

\section{Table 3. Fuzzy values}

\begin{tabular}{|l|l|}
\hline Trans_Id & Level-1 Fuzzy set \\
\hline T1 & $\left(\frac{0.8}{1 . \text { low }}\right)\left(\frac{0.7}{2 . m i d d l e}+\frac{0.3}{2 . \text { high }}\right)$ \\
\hline T2 & $\left(\frac{0.4}{1 . \text { middle }}+\frac{0.6}{1 . \text { high }}\right)\left(\frac{0.1}{2 . m i d d l e}+\frac{0.9}{2 . \text { high }}\right)$ \\
\hline T3 & $\left(\frac{0.9}{1 . \text { middle }}+\frac{0.1}{1 . \text { high }}\right)\left(\frac{0.9}{2 . \text { high }}\right)$ \\
\hline T4 & $\left(\frac{0.4}{1 . \text { low }}+\frac{0.6}{1 . \text { middle }}\right)\left(\frac{0.8}{2 . h i g h}\right)$ \\
\hline T5 & $\left(\frac{0.6}{1 . m i d d l e}+\frac{0.4}{1 . \text { high }}\right)\left(\frac{0.1}{2 . \text { high }}\right)$ \\
\hline T6 & $\left(\frac{0.1}{1 . \text { middle }}+\frac{0.9}{1 . h i g h}\right)\left(\frac{0.1}{2 . h i g h}\right)$ \\
\hline
\end{tabular}

Step 5: The counts of fuzzy set are in table 4 .

Table 4. Fuzzy Count at level-1

\begin{tabular}{|l|l|l|l|}
\hline Item & Count & Item & Count \\
\hline 1.low & 1.2 & 2.middle & 0.8 \\
\hline 1.middle & 2.6 & 2.high & 2.5 \\
\hline 1.high & 2.0 & & \\
\hline
\end{tabular}

Step 6: Compare the count of each item with the minimum support threshold i.e. $\alpha_{1}^{1}=1.8$ and put this Set of large 1 itemsets in table 5 .

Table 5.Fuzzy Count inL1
\begin{tabular}{|l|l|}
\hline Itemset & Count \\
\hline 1.middle & 2.6 \\
\hline 1.high & 2.0 \\
\hline 2.high & 2.5 \\
\hline
\end{tabular}

Step 7: Candidate set $C_{2}$ is generated from $L_{1}$.

Step 8: The following sub steps are done for each newly formed candidate 2-itemset in $\mathrm{C}_{2}$

(a)The fuzzy membership values of each transaction data for the candidate -2 itemsets are calculated. Minimum operator is used to find the membership value.

(b)The scalar cardinality (count) of each candidate 2 itemset is calculated which are shown in table 6. (c) Since only the count of (1.middle,2.high) is greater than $\alpha_{2}^{1}$ or 1.5 ,it is thus stored in $\mathrm{L}_{2}$

Table 6.The fuzzy counts of the itemsets in $\mathrm{C}_{2}$

\begin{tabular}{|l|l|}
\hline Itemset & Count \\
\hline (1.middle,1.high) & 1.0 \\
\hline (1.middle,2.high) & 1.5 \\
\hline (1.high,2.high) & 0.9 \\
\hline
\end{tabular}

Step $9,10: r$ is set to 2 .The candidate set $C_{3}$ is generated from $\mathrm{L}_{2}$. Since it is Null, step 14 is executed to find association rules.

Step 14: Possible association rule is:

If $1=$ middle, then $2=$ high.

The confidence value is calculated by using the below equation:- 


$$
\frac{\sum_{i=1}^{6}(1 . \text { middlen2.high })}{\sum_{i=1}^{6}(1 . \text { middle })}=\frac{1.5}{2.6}=\mathbf{0 . 5 8} .
$$

Step 15: Since the confidence value of this rule is greater than $\beta$ or 0.55 , hence it is strong rule.

Step 16: Set $L=2$ for the next level do the same process. Now following are the tables for level 2 .

Table 7. Fuzzy values at level 2

\begin{tabular}{|c|c|}
\hline $\begin{array}{l}\text { Trans- } \\
\text { ID }\end{array}$ & Level-2 Fuzzy set \\
\hline T1 & $\begin{array}{l}\left(\frac{1.0}{11 . l o w}\right)\left(\frac{0.6}{12 . l o w}+\right. \\
\left.\frac{0.09}{12 . m i d d l e}\right)\left(\frac{0.6}{21 . l o w}\right)\left(\frac{1.0}{22 . l o w}\right)\left(\frac{0.5}{23 . m i d d l e}\right)\end{array}$ \\
\hline $\mathrm{T} 2$ & 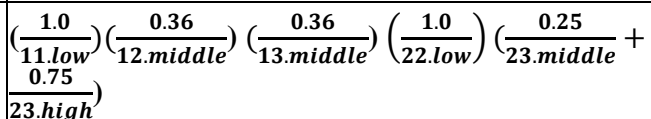 \\
\hline T3 & $\begin{array}{l}\left(\frac{0.6}{11 . l o w}\right)\left(\frac{0.63}{12 . m i d d l e}\right)\left(\frac{0.33}{21 . l o w}+\right. \\
\left.\frac{0.18}{21 . m i d d l e}\right)\left(\frac{0.6}{22 . l o w}\right)\left(\frac{0.25}{23 . m i d d l e}+\frac{0.75}{23 . h i g h}\right)\end{array}$ \\
\hline $\mathrm{T} 4$ & $\left(\frac{0.54}{12 . m i d d l e}\right)\left(\frac{0.63}{21 . \text { middle }}\right)\left(\frac{0.25}{23 . \text { high }}\right)$ \\
\hline T5 & $\left(\begin{array}{l}\frac{0.36}{11 . m i d d l e} \\
\left.\frac{0.25}{23 . \text { high }}\right)\end{array}\right)\left(\frac{0.45}{13 . \text { middle }}\right)\left(\frac{0.36}{22 . m i d d l e}\right)\left(\frac{0.75}{23 . \text { middle }}+\right.$ \\
\hline T6 & $\left(\frac{0.6}{11 . l o w}\right)\left(\frac{0.45}{12 . m i d d l e}\right)\left(\frac{0.65}{13 . m i d d l e}\right)\left(\frac{0.6}{22 . l o w}\right)\left(\frac{0.5}{23 . h i g h}\right)$ \\
\hline
\end{tabular}

Table-8. Values of Fuzzy count at level-2

\begin{tabular}{|l|l|l|l|}
\hline Item & Count & Item & Count \\
\hline 11.low & 3.2 & 21.low & 0.93 \\
\hline 11.middle & 0.36 & 21.middle & 0.81 \\
\hline 11.high & 0.0 & 21.high & 0.0 \\
\hline 12.low & 0.6 & 22.low & 3.2 \\
\hline 12.middle & 2.07 & 22.middle & 0.36 \\
\hline 12.high & 0.0 & 22.high & 0.0 \\
\hline 13.low & 0.0 & 23.low & 0.0 \\
\hline 13.middle & 1.46 & 23.middle & 1.75 \\
\hline 13.high & 0.0 & 23.high & 2.7 \\
\hline
\end{tabular}

Compare the count of each item with the minimum support threshold $\alpha_{1}^{2}=1.6$ and put this item in table 9 .

Table 9.Fuzzy count at level-2

\begin{tabular}{|l|l|}
\hline Itemset & Count \\
\hline 11. low & 3.2 \\
\hline
\end{tabular}

\begin{tabular}{|l|l|}
\hline 12.middle & 2.07 \\
\hline 22.low & 3.2 \\
\hline 23.middle & 1.75 \\
\hline 23.high & 2.7 \\
\hline
\end{tabular}

Table 10.The fuzzy counts of the itemsets in $\mathrm{C}_{2}$

\begin{tabular}{|l|l|l|l|}
\hline Itemset & Count & Itemset & Count \\
\hline (11.low,12.middle) & 1.56 & (12.middle,22.low) & 1.56 \\
\hline (11.low,22.low) & 3.2 & (12.middle,23.middle) & 0.59 \\
\hline (11.low,23.middle) & 1.0 & (12.middle,23.high) & 1.69 \\
\hline (11.low,23.high) & 1.85 & (22.low,23.middle) & 1.0 \\
\hline (22.low,23.high) & 1.85 & (23.middle,23.high) & 0.75 \\
\hline
\end{tabular}

Compare the count of each item with $\alpha_{2}^{2}=1.55$ and put this Set of large 2 itemsets in $\mathrm{C}_{3}$.

Table 11.The fuzzy counts of the itemsets in $\mathrm{C}_{3}$

\begin{tabular}{|l|l|l|l|}
\hline Itemset & Count & Itemset & Count \\
\hline (11.low,12.middle) & 1.56 & (12.middle,22.low) & 1.56 \\
\hline$(11$. low,22.low) & 3.2 & (12.middle,23.high) & 1.69 \\
\hline (11.low,23.high) & 1.85 & (22.low,23.high) & 1.85 \\
\hline
\end{tabular}

Table 12. Membership values $L_{3}$

\begin{tabular}{|l|l|}
\hline Itemset & Count \\
\hline (11.low,12.middle,22.low) & 1.5 \\
\hline (11.low,12.middle,23.high) & 1.41 \\
\hline (11.low,22.low,23.high) & 1.85 \\
\hline (12.middle,22.low,23.high) & 1.5 \\
\hline
\end{tabular}

Compare the count of each item with $\alpha^{2}{ }_{3} 1.54$ and put this Set of large 3 itemsets in $\mathrm{C}_{4}$.

Now we will calculate $\mathrm{L}_{4}$, since this is Null, so we will not go further .

Table 13. Association rules at level -2 with confidence values.

\begin{tabular}{|l|l|}
\hline Association Rule & Confidence \\
\hline (11.low) => (22.low, 23.high) & 0.58 \\
\hline (11.low, 22.low) => (23.high) & 0.84 \\
\hline (11.low, 23.high) => (22.low) & $(0.58$ \\
\hline (22.low) => (11.low, 23.high) & 0.58 \\
\hline (22.low, 23.high) => (11.low) & 1.73 \\
\hline (23.high) => (11.low, 22.low) & 1.18 \\
\hline
\end{tabular}

By repeating the same step the following rules are generated for level 3. 
Table 14. Association rules at level -3 with confidence values

\begin{tabular}{|l|l|}
\hline Association Rule & Confidence \\
\hline (112.low)=> 232.middle) & 0.73 \\
\hline (232.middle) $=>$ (112.low) & 0.57 \\
\hline (121.middle) $=>$ (232.middle) & $(0.62$ \\
\hline (232.middle) $=>$ (121.middle) & 0.57 \\
\hline (221.low) => (232.middle) & 0.8 \\
\hline (232.middle) => (221.low) & 0.57 \\
\hline (231.high) => (232.middle) & 0.67 \\
\hline (232.middle) => (231.high) & 0.86 \\
\hline
\end{tabular}

\section{EXPERIMENTAL RESULTS}

In this part, we will analyze the results of the experiments and analyses made. They are implemented in JAVA using NETBEAN and Jdk1.6.0. The proposed algorithm carries out the analysis on a number of 200 sales invoices of a seed store and 7 of its items are based on the predefined taxonomy from 7 items and the predefined membership function per each item, carries out the mining of association rules. The predefined taxonomy in the first level includes 5 nodes that represent the items used in the test, the second level includes 10 nodes that represent the name of different seeds and in the third level it also consists of 34 nodes that represent the manufacturing companies.

Each transaction includes the name of the product purchased and also the quantity of that product. One item may not be used twice in one transaction. Experiments were first made to show the relationships between numbers of rules mined and minimum support values along with different minimum confidence values.

In figure 4 we can see that with the increment in number of the transactions, the number of mined association rules will also be increasing, and this is obvious, because with the increased number of the transactions, the number of frequent itemset will also increase and as a result, a greater number of rules are mined.

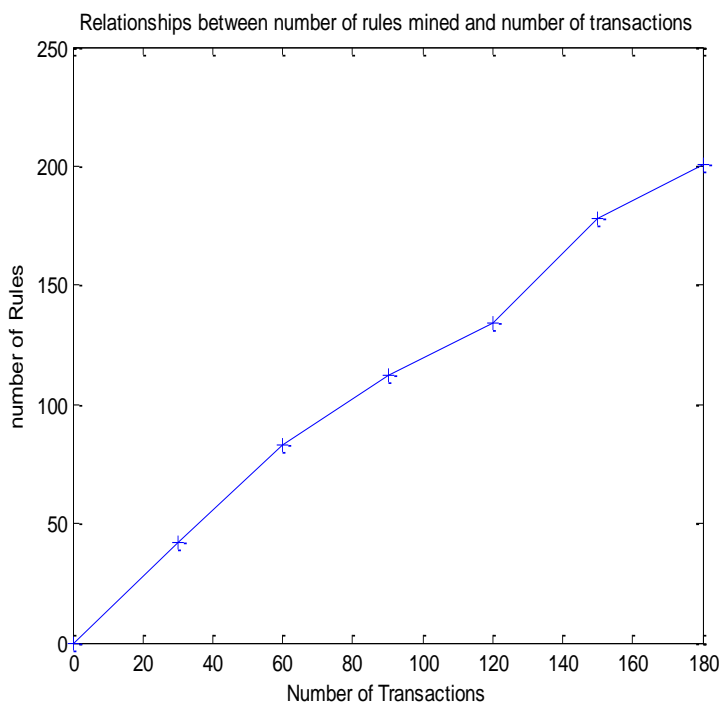

Fig. 4: Relationship between number of transactions and rules

The results obtained based on the number of rules developed and different types of the predefined minimum confidence by the user have been shown in Figure 5 based on the 150 transactions of the customers' purchases and minimum support equal to 1.5 .

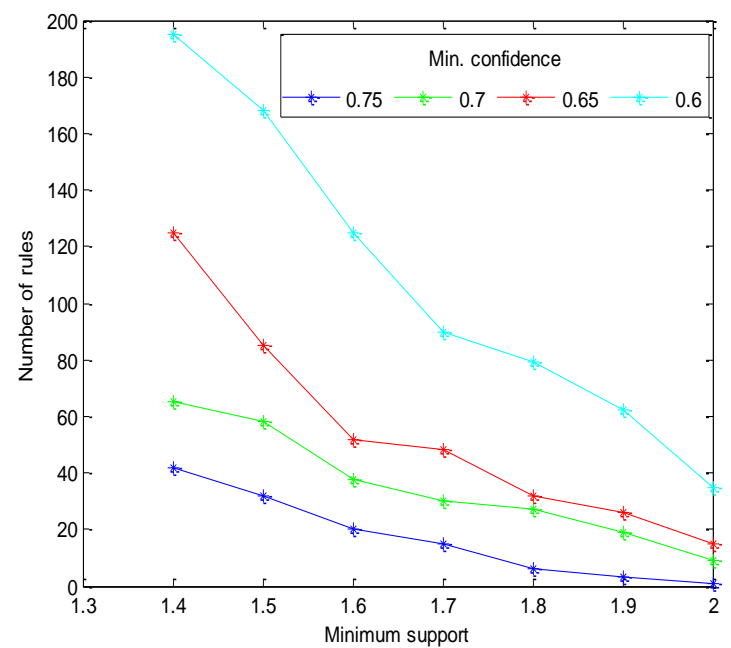

Fig. 5: Relationship between number of rules generated and minimum support and confidence

From Fig. 5, it is easily seen that numbers of rules mined decrease along with increase of minimum support values. This is quite consistent with our intuition. Also, the curves from larger minimum confidence values are smoother than those from smaller minimum confidence values, meaning that the minimum support values have a larger effect on the numbers of rules mined from smaller minimum confidence values.

\section{CONCLUSION AND FUTURE SCOPE}

In this paper, a hierarchical fuzzy association rule mining algorithm for extracting implicit knowledge from multi-level dataset is purposed. It integrates fuzzy set concepts, datamining technologies and multiple-level taxonomy to find hierarchical fuzzy association rules from transaction data sets. The objective of the proposed work is to get rules which are optimal with respect to the two criteria- 1) generate more specific and concrete information and 2) maximizing the accuracy of rule. The proposed method has got the ability to mine association rules at different levels based on the user's tendency. As a result the mined rules can be more close to the user's demand. As an example, the users may have tendency that the mined rules should only include the items defined at the first level, so for other levels, except the first level, they can consider a big value of the minimum support so that the frequent itemset is not mined from those levels and as a result no rules are derived in those levels.

Although the proposed method works well in data mining for quantitative values, it is just a beginning. There is still much work to be done in this field. In our method we are taking only positive association rules i.e. $\mathrm{A}=>\mathrm{B}$, in our next study we will also take negative association rules like $\ulcorner\mathrm{A}=>-\mathrm{B}, \mathrm{A}=>-\mathrm{B}$ and $-\mathrm{A}=>\mathrm{B}$.

\section{REFERENCES}

[1] Han J., Kamber M. 2001. Data Mining: Concepts and Techniques.The Morgan Kaufmann Series.

[2] Agrawal R., Imielinksi T. and A. Swami. 1993. Mining association rules between sets of items in large database, ACM SIGMOD Conference, Washington, DC, USA 207-216. 
[3] Agrawal R., Imielinksi T. and A. Swami. 1993. Database mining: a performance perspective, IEEE Trans. Knowledge Data Eng. 5 (6) 914-925.

[4] Chen M.S., Han J. and Yu P.S. 199., Data mining: an overview from a database perspective, IEEE Trans. Knowledge Data Eng. 8 (6) (1996) 866-883.

[5] Famili, A., Shen, W.M., Weber, R.,and Simoudis, E. 1997. Data preprocessing and intelligent data analysis, Intel. Data Anal.1 (1), 1-28.

[6] Hong T.P., Kuo C.S. and Chi S.C. 1999. A data mining algorithm for transaction data with quantitative values, Intell. Data Anal. 3 (5). 363-376.

[7] Srikant R. and Agrawal R. 1996. Mining quantitative association rules in large relational tables, The ACM SIGMOD International Conference on Management of Data, Monreal, Canada, pp. 1-12.

[8] Zadeh L.A. 1965. Fuzzy sets, Inform. and Control 8 (3) 338-353.

[9] Kandel A. 1992. Fuzzy Expert Systems, CRC Press, Boca Raton, FL. pp. 8-19.

[10] Ha and Fu Y. 1999. Mining Multiple - Level Association Rules in Large Databases, IEEE TKDE. 1, pp. 798 - 805.

[11] Srikant R. and Agrawal R. 1995. Mining generalized association rules, The International Conference on Very Large Databases.

[12] Frawley, W.J., Piatetsky-Sapiro, G., and Matheus, C.J. 1992.Knowledge discovery in databases: An overview, International Journal of Computer Theory and Engineering, Vol. 3, No. 2,

[13] Ishibuchi, H., Yamamoto, T. and Nakashima, T. 2001. ICDM Proceedings of the IEEE International Conference on Data Mining. pp. 241-248.

[14] Han, J., and Fu, Y. 1995. Discovery of Multiple-level Association Rules from Large Databases.

[15] Srikant, R., and Agrawal R. 1995. Mining Generalized Association Rules, In Proceedings of the 21st VLDB Conference, Zurich, Switzerland.
[16] Srikant R. and Agarwal R. 1996. Mining quantitative association rules in large relational tables, In Proceedings of the ACM SIGMOD International Conference on Management of Data, pp 1-12, Montreal,Quebec, Canada.

[17] Kuok, C.H., Fu, A., and Wong, M.H. 1998. Mining Fuzzy association rules in databases ACM SIGMOD Record, 27(1), ACM Press.

[18] Chen, G., Wei, Q., and Kerre E. 2000. Fuzzy Data Mining: Discovery of Fuzzy Generalized Association Rules. In Proceedings of Recent Research issues on Management of Fuzziness in Databases, Physica-verlag (Springer).

[19] Lee J. H. and Kwang H. L. 1997. An extension of association rules using fuzzy sets, presented at the IFSA'97, Prague, Czech Republic.

[20] Gautam Pratima and Pardasani K.R.. 2010. A Novel Approach For Discovery Multi Level Fuzzy Association Rule Mining, Journal of computing, volume 2, issue 3 , march , ISSN 2151-9617.

[21] Koh Y.S., Rountree N. and O'Keefe R.A 2006 Finding Non-Coincidental Sporadic Rules Using Apriori-Inverse Int'l J. Data Warehousing and Mining, vol. 2, pp. 38-54.

[22] Usha Rani, Vijaya Prakash R, and. Govardhan A. Mining Multi Level Association Rules Using Fuzzy Logic International Journal of Emerging Technology and Advanced Engineering August 2013.

[23] Ying Lin, K., Chian B. ,Chian and T. Pei Hong, 2003. Mining Fuzzy Multiple-Level Association Rules from Quantitative Data. Applied Intelligence, 18: 79-90. Kluwer Academic Publishers. Proceedings of the $21^{\text {st }}$ International Conference on VLDB, Zurich, Switzerland.

[24] Prakash, S., Vijayakumar M., and. Parvathi R.M.S A Novel Method of Mining Association Rule with Multi Level Concept Hierarchy, International Conference on Advanced Computer Technology (ICACT) Proceedings published by International Journal of Computer Applications (IJCA)2011. 